\title{
Leader-Following Consensus of Fractional Nonlinear Multiagent Systems
}

\author{
Guojian Ren, Yongguang Yu, and Shuo Zhang \\ Department of Mathematics, Beijing Jiaotong University, Beijing 100044, China \\ Correspondence should be addressed to Yongguang Yu; ygyu@bjtu.edu.cn
}

Received 23 February 2015; Accepted 30 May 2015

Academic Editor: Ciprian G. Gal

Copyright ( 2015 Guojian Ren et al. This is an open access article distributed under the Creative Commons Attribution License, which permits unrestricted use, distribution, and reproduction in any medium, provided the original work is properly cited.

\begin{abstract}
The leader-following consensus of fractional nonlinear multiagent systems is investigated over an undirected fixed interaction graph. Mittag-Leffler stability and the fractional Lyapunov direct method are firstly introduced into the fractional multiagent systems. The sufficient conditions are given to guarantee that the leader-following consensus can be achieved in the systems with both single-integrator dynamics and double-integrator dynamics. Finally, the numerical simulations are given to verify the correctness of the presented theory.
\end{abstract}

\section{Introduction}

Since the precursory works of Reynolds [1] and Vicsek et al. [2], the consensus problem of multiagent systems has become a hot topic in the engineering community. It has been applied to the analysis of animal group behaviors, the formation control of unmanned air vehicles, and the distributed coordination of sensor networks. So, due to its broad applications, the consensus problem has been more and more widely studied in various systems, such as biological systems and social and engineering systems. For a cooperative multiagent system, leaderless consensus means that all agents reach an agreement on certain global criteria of common interest by sharing information locally with their neighbors, which has been widely studied in the integer-order systems with both single-integrator dynamics and double-integrator dynamics [3-6], while leader-following consensus means that there exists a virtual leader which specifies an objective for all agents to follow.

In the past few years, the leader-following consensus problem of integer-order multiagent systems has been intensively studied. In [7], the leader-following consensus problem for second-order multiagent systems with nonlinear inherent dynamics was investigated and the distributed control protocols were proposed under both fixed undirected and fixed directed communication topologies. In [8], the secondorder leader-following consensus problem of nonlinear multiagent systems was studied with general network topologies. Moreover the leaderless and leader-following consensus algorithms with communication and input delays under a directed network topology were considered in [9].

At present, a growing number of works by many authors from various fields of science and engineering have been dealing with dynamical systems described by fractional equations. Fractional calculus is a generalization of integer-order differentiation and integration to arbitrary noninteger order, which provides a powerful instrument for the description of memory and hereditary properties of different substances. In recent years, more and more researchers mainly pay attention to the distributed coordination for multiagent systems with fractional dynamics. In [10], Cao et al. studied the coordination algorithms for networked fractional systems with singleintegrator dynamics and obtained the sufficient conditions such that the distributed coordination can be achieved. Yang et al. [11] investigated the distributed coordination of fractional multiagent systems with communication delays. And Cao and Ren [12] discussed the distributed formation control problem for fractional multiagent systems under dynamic interaction and with absolute/relative damping.

Nonlinear dynamics can be used to describe many complex phenomena in natural world and human society. Hence, it is more practical to investigate the collective behavior of the fractional nonlinear multiagent systems. Motivated by the above discussion, we consider fractional nonlinear 
multiagent systems in this paper. First, we study the leaderfollowing consensus in the fractional systems with both single-integrator dynamics and double-integrator dynamics, respectively. Second, we obtain the sufficient conditions to ensure the leader-following consensus by introducing MittagLeffler stability and the fractional Lyapunov direct method into fractional multiagent systems. Finally, we give some numerical simulations to illustrate the correctness of the presented results.

The rest of this paper is arranged as follows. The graph theory notions and some definitions of fractional calculus are introduced as a basis in Section 2. The main results on leaderfollowing consensus for fractional nonlinear multiagent systems are presented in Sections 3 and 4. Then, some simulation results are given in Section 5. Finally, a short conclusion is shown to close the paper in Section 6.

\section{Preliminaries}

In this section, some basic notions about the graph theory and some definitions of fractional calculus are introduced as the preliminaries of this paper. And some necessary conclusions are presented for the use of next several sections.

2.1. Graph Theory Notions. The interaction topologies in the multiagent systems consisting of $n$ agents can be modelled by a graph $\mathscr{G}=(\mathscr{V}, \mathscr{W})$, where $\mathscr{V}=\left\{v_{1}, v_{2}, \ldots, v_{n}\right\}$ and $\mathscr{W} \subseteq \mathscr{V}^{2}$ represent the agent set and the edge set of the graph, respectively. Each edge denoted by $\left(v_{i}, v_{j}\right)$ means that agent $j$ can access the state information of agent $i$. Accordingly, agent $i$ is a neighbor of agent $j$. All neighbors of agent $i$ are denoted by $N_{i}$. Then, we introduce two types of matrices to represent the interaction graph: (1) the adjacency matrix $A=\left[a_{i j}\right] \epsilon$ $R^{n \times n}$ with $a_{i j}=1$ if $\left(v_{i}, v_{j}\right) \in \mathscr{W}$ and $a_{i j}=0$; otherwise, (2) the (nonsymmetric) Laplacian matrix $L=\left[l_{i j}\right] \in R^{n \times n}$ with $l_{i i}=\sum_{j \in N_{i}} a_{i j}$ and $l_{i j}=-a_{i j}, i \neq j$.

Lemma 1 (see [13]). Suppose that the connection topology of $n$ agents is undirected and connected. The matrix $L+$ $B$ is symmetric positive definite, where $L$ is the Laplacian matrix of the connection topology of $n$ agents and $B=$ $\operatorname{diag}\left\{b_{1}, b_{2}, \ldots, b_{n}\right\}, i=1,2, \ldots, n$, where $b_{i}$ is a nonnegative real number that does not always equal 0 .

2.2. Caputo Fractional Operator and Mittag-Leffler Stability. There are mainly two widely used fractional operators: Caputo and Riemann-Liouville (R-L) fractional operators [14]. In this paper, the Caputo fractional operator is used because the Caputo fractional derivative owns the same initial conditions with integer-order derivative, which is well understood in physical situations and more applicable to realworld problems.

Definition 2 (see [15]). Caputo's fractional derivative of order $\alpha$ for a function $f \in C^{n}\left(\left[t_{0},+\infty\right], R\right)$ is defined by

$$
{ }_{t_{0}} D_{t}^{\alpha} f(t)=\frac{1}{\Gamma(n-\alpha)} \int_{t_{0}}^{t} \frac{f^{(n)}(\tau)}{(t-\tau)^{\alpha-n+1}} d \tau,
$$

where $t \geq t_{0}$ and $n$ is a positive integer such that $n-1<\alpha<n$. Particularly, when $0<\alpha<1$,

$$
{ }_{t_{0}} D_{t}^{\alpha} f(t)=\frac{1}{\Gamma(1-\alpha)} \int_{t_{0}}^{t} \frac{f^{\prime}(\tau)}{(t-\tau)^{\alpha}} d \tau .
$$

In the following, we will introduce the Mittag-Leffler function [14]. For $\alpha, \beta \in C$, the Mittag-Leffler function in two parameters is defined as

$$
E_{\alpha, \beta}(z)=\sum_{k=0}^{\infty} \frac{z^{k}}{\Gamma(k a+\beta)} .
$$

When $\beta=1$ and $\alpha>0$, (3) can be written in a special case as $E_{\alpha}(z)=\sum_{k=0}^{\infty}\left(z^{k} / \Gamma(k a+1)\right)$.

As an important dynamical property of fractional systems, Mittag-Leffler stability is introduced in the following. Consider the following $n$-dimensional Caputo fractionalorder system:

$$
\begin{aligned}
t_{0} D_{t}^{\alpha} x(t) & =f(t, x(t)), \\
x\left(t_{0}\right) & =x_{t_{0}},
\end{aligned}
$$

where $\alpha \in(0,1), x=\left(x_{1}, x_{2}, \ldots, x_{n}\right)^{T} \in R^{n}, t_{0} \geq 0$, and $f$ is piecewise continuous on $t$ and satisfies locally Lipschitz condition on $x$.

Assumption 3. The nonlinear function $f(t, x, y)(x, y \in R)$ is continuous and satisfies Lipschitz condition on $x$ and $y$ with Lipschitz constant $l \geq 0$; that is,

$$
|f(t, x, y)-f(t, u, v)| \leq l(|x-u|+|y-v|)
$$

for any $t \geq 0$.

Definition 4 (see [16]). The constant $\bar{x}$ is an equilibrium point of Caputo fractional-order dynamic system (4) if and only if $f(t, \bar{x})=0$.

Definition 5 (see [16]). If $\bar{x}=0$ is an equilibrium point of system (4), the solution of (4) is said to be Mittag-Leffler stable if

$$
\|x(t)\| \leq\left[m\left(x_{t_{0}}\right) E_{\alpha}\left(-\lambda\left(t-t_{0}\right)^{\alpha}\right)\right]^{b}
$$

where $\lambda>0, b>0, m(0)=0,\|\cdot\|$ denotes an arbitrary norm, and $m(x) \geq 0$ satisfies locally Lipschitz condition on $x \in R^{n}$ with Lipschitz constant $m_{0}$.

Remark 6. Mittag-Leffler stability for system (4) implies asymptotic stability for any initial value; that is, $\|x\| \rightarrow 0$ with $t \rightarrow+\infty$.

Lemma 7 (see [15]). There exists a unique solution of system (4) for any initial value, if system (4) has equilibrium point at the origin and $f(t, x)$ satisfies locally Lipschitz condition on $x$. 
Lemma 8 (see [17]). When $t_{0}=0$, the fractional-order system (4) is Mittag-Leffler stable at the equilibrium point $\bar{x}=0$, if there exists a continuous function $V(t, x(t))$ which satisfies

$$
\begin{aligned}
& \alpha_{1}\|x\|^{a} \leq V(t, x(t)) \leq \alpha_{2}\|x\|^{a b}, \\
& { }_{0} D_{t}^{\beta} V\left(t^{+}, x\left(t^{+}\right)\right) \\
& \quad \leq-\alpha_{3}\|x(t)\|^{a b} \text { (holding almost everywhere), }
\end{aligned}
$$

where $V(t, x(t)):[0, \infty) \times D \rightarrow R$ satisfies locally Lipschitz condition on $x ; \dot{V}(t, x(t))$ is piecewise continuous and $\lim _{\tau \rightarrow t^{+}} \dot{V}(\tau, x(\tau))$ exists for any $t \in[0, \infty) ; D \subset$ $R^{n}$ is a domain containing the origin and $V\left(t^{+}, x\left(t^{+}\right)\right) \triangleq$ $\lim _{\tau \rightarrow t^{+}} V(\tau, x(\tau))$; and $t \geq 0, \beta \in(0,1)$, and $\alpha_{1}, \alpha_{2}, \alpha_{3}, a$, and $b$ are arbitrary positive constants. If the assumptions hold globally on $R^{n}$, then $\bar{x}=0$ is globally Mittag-Leffler stable.

Lemma 9 (see [17]). If $h(t) \in C^{1}([0,+\infty), R)$ denotes a continuously differentiable function, the following inequality holds almost everywhere:

$$
{ }_{0} D_{t}^{\alpha}\left|h\left(t^{+}\right)\right| \leq \operatorname{sgn}(h(t)){ }_{0} D_{t}^{\alpha} h(t), \quad 0<\alpha<1,
$$

where $h\left(t^{+}\right) \triangleq \lim _{\tau \rightarrow t^{+}} h(\tau)$.

\section{Leader-Following Consensus for Single-Integrator Dynamics}

In this section, the leader-following consensus for fractional multiagent systems with single-integrator dynamics is studied, and the sufficient condition is obtained to achieve the leader-following consensus over an undirected fixed interaction graph.

Consider the multiagent system consisting of $n$ agents, labeled as agents 1 to $n$. The single-integrator dynamics of each agent is given by

$$
{ }_{0} D_{t}^{\alpha} x_{i}(t)=f\left(t, x_{i}(t)\right)+u_{i}(t), \quad i \in\{1,2, \ldots, n\},
$$

where $\alpha \in(0,1), x_{i}(t)$ is the state for the $i$ th agent, $u_{i}(t)$ is the control input for the $i$ th agent, and $f\left(t, x_{i}(t)\right)$ is the intrinsic nonlinear dynamics for the $i$ th agent.

The virtual leader for multiagent system (10) is an isolated agent described by

$$
{ }_{0} D_{t}^{\alpha} x_{r}(t)=f\left(t, x_{r}(t)\right)
$$

where $x_{r}(t)$ is the state for the virtual leader.

In the following, assume that all agents are in a onedimensional space for the simplicity of presentation. However, all results hereafter are still valid for the $m$-dimensional $(m>1)$ space by the introduction of the Kronecker product.

Definition 10. The multiagent system (10) is said to achieve leader-following consensus if its solution satisfies $\lim _{t \rightarrow+\infty}\left\|x_{i}(t)-x_{r}(t)\right\|=0, i=1,2, \ldots, n$, for any initial condition.
The control protocol is proposed as

$$
\begin{aligned}
& u_{i}(t) \\
& \quad=-\beta\left[\sum_{j=1}^{n} a_{i j}\left(x_{i}(t)-x_{j}(t)\right)+b_{i}\left(x_{i}(t)-x_{r}(t)\right)\right],
\end{aligned}
$$

where $a_{i j}(i, j=1,2, \ldots, n)$ is the $(i, j)$ th entry of the adjacency matrix $A \in R^{n \times n}$ associated with the undirected graph $\mathscr{G} ; b_{i}(i=1,2, \ldots, n)$ is a nonnegative real number that does not always equal 0 ; and $\beta$ is a nonnegative constant.

Let $B=\operatorname{diag}\left\{b_{1}, b_{2}, \ldots, b_{n}\right\}$ and $H=L+B$, where $L$ is the Laplacian matrix of $\mathscr{G}$. Let matrix $\Lambda$ be the Jordan canonical form of matrix $H$, which satisfies $\Lambda=P^{-1} H P$, where $P=$ $\left[p_{i j}\right] \in R^{n \times n}$ and $P^{-1}=\left[\overline{p_{i j}}\right] \in R^{n \times n}$.

In the rest of this paper, $\|x\|=\left|x_{1}\right|+\left|x_{2}\right|+\cdots+\left|x_{n}\right|=$ $\sum_{i=1}^{n}\left|x_{i}\right|$, where $x$ is a vector and $x=\left[x_{1}, x_{2}, \ldots, x_{n}\right]^{T}$, and $\|B\|_{1}=\max _{1 \leq j \leq n}\left\{\sum_{i=1}^{n}\left|b_{i j}\right|\right\}$, where $B$ is a matrix and $B=$ $\left[b_{i j}\right] \in R^{n \times n}$.

Theorem 11. Suppose that the fixed undirected graph $\mathscr{G}$ is connected and the nonlinear function $f$ in systems (10) and (11) satisfies Assumption 3. Using the control input (12) for system (10), if $\beta / l \geq\|P\|_{1}\left\|P^{-1}\right\|_{1} / \min _{1 \leq i \leq n}\left\{\lambda_{i}\right\}$, where $\lambda_{i}$ is the ith eigenvalue of the matrix $H, i \in\{1,2, \ldots, n\}$, then $\mid x_{i}(t)-$ $x_{r}(t) \mid \rightarrow 0$, as $t \rightarrow+\infty$, which implies that the multiagent system (10) is said to achieve leader-following consensus.

Proof. With the control input (12), system (10) can be rewritten as

$$
\begin{aligned}
& { }_{0} D_{t}^{\alpha} x_{i}(t) \\
& =f\left(t, x_{i}(t)\right) \\
& \quad-\beta\left[\sum_{j=1}^{n} a_{i j}\left(x_{i}(t)-x_{j}(t)\right)+d_{i}\left(x_{i}(t)-x_{r}(t)\right)\right] .
\end{aligned}
$$

Noting that $y_{i}(t)=x_{i}(t)-x_{r}(t), i=1,2, \ldots, n$, we have

$$
\begin{aligned}
{ }_{0} D_{t}^{\alpha} y_{i}(t)= & f\left(t, y_{i}(t)+x_{r}(t)\right)-f\left(t, x_{r}(t)\right) \\
& -\beta\left[\sum_{j=1}^{n} a_{i j}\left(y_{i}(t)-y_{j}(t)\right)+d_{i} y_{i}(t)\right],
\end{aligned}
$$

which can be written in a vector form as

$$
{ }_{0} D_{t}^{\alpha} Y(t)=-\beta H Y(t)+F(t, Y(t))
$$

where $Y(t)=\left[y_{1}(t), y_{2}(t), \ldots, y_{n}(t)\right]^{T}, F(t, Y(t))=[f(t$, $\left.y_{1}(t)+x_{r}(t)\right)-f\left(t, x_{r}(t)\right), f\left(t, y_{2}(t)+x_{r}(t)\right)-f\left(t, x_{r}(t)\right)$, $\left.\ldots, f\left(t, y_{n}(t)+x_{r}(t)\right)-f\left(t, x_{r}(t)\right)\right]^{T}$.

Let $Z(t)=P^{-1} Y(t)$, and (15) can be rewritten as

$$
{ }_{0} D_{t}^{\alpha} Z(t)=-\beta \Lambda Z(t)+P^{-1} F(t, P Z(t)),
$$


where $Z(t)=\left[z_{1}(t), z_{2}(t), \ldots, z_{n}(t)\right]^{T}, F(t, P Z(t))=$ $\left[f\left(t, \sum_{k=1}^{n} p_{1 k} z_{k}(t)+x_{r}(t)\right)-f\left(t, x_{r}(t)\right), f\left(t, \sum_{k=1}^{n} p_{2 k} z_{k}(t)+\right.\right.$ $\left.x_{r}(t)\right)-f\left(t, x_{r}(t)\right), \ldots, f\left(t, \sum_{k=1}^{n} p_{n k} z_{k}(t)+x_{r}(t)\right)-$ $\left.f\left(t, x_{r}(t)\right)\right]^{T}$.

Because the fixed undirected graph $\mathscr{G}$ is connected and $b_{i}(i=1,2, \ldots, n)$ is a nonnegative real number that does not always equal 0 , from Lemma $1, H$ is symmetric positive definite and $\Lambda$ is a diagonal positive definite matrix; that is, $\Lambda=\operatorname{diag}\left\{\lambda_{1}, \lambda_{2}, \ldots, \lambda_{n}\right\}, \lambda_{i}>0, i=1,2, \ldots, n$. From Lemma 7 and Assumption 3, system (16) has a unique and continuous solution $Z(t)$. In what follows, we demonstrate that system (16) is asymptotically stable.

Consider the Lyapunov function candidate constructed as

$$
V(t, z(t))=\sum_{i=1}^{n}\left|z_{i}(t)\right| .
$$

Obviously, the Lyapunov functional (17) satisfies inequality (7). According to Lemma 9, the following inequality holds almost everywhere:

$$
\begin{aligned}
& { }_{0} D_{t}^{\alpha} V\left(t^{+}, z\left(t^{+}\right)\right)=\sum_{i=1}^{n}{ }_{0} D_{t}^{\alpha}\left|z_{i}\left(t^{+}\right)\right| \leq \sum_{i=1}^{n} \operatorname{sgn}\left(z_{i}(t)\right) \\
& { }_{0} D_{t}^{\alpha} z_{i}(t)=\sum_{i=1}^{n} \operatorname{sgn}\left(z_{i}(t)\right)\left[-\beta \lambda_{i} z_{i}(t)\right. \\
& \left.+\sum_{j=1}^{n} \overline{p_{i j}}\left(f\left(t, \sum_{k=1}^{n} p_{j k} z_{k}(t)+x_{r}(t)\right)-f\left(t, x_{r}(t)\right)\right)\right] \\
& \leq \sum_{i=1}^{n}\left[-\beta \lambda_{i}\left|z_{i}(t)\right|+l \sum_{j=1}^{n}\left|\overline{p_{i j}}\right|\left|\sum_{k=1}^{n} p_{j k} z_{k}(t)\right|\right] \\
& \leq \sum_{i=1}^{n}\left[-\beta \lambda_{i}\left|z_{i}(t)\right|+l \sum_{j=1}^{n}\left|\overline{p_{i j}}\right| \sum_{k=1}^{n}\left|p_{j k}\right|\left|z_{k}(t)\right|\right] \\
& =-\beta \sum_{i=1}^{n} \lambda_{i}\left|z_{i}(t)\right|+l \sum_{i=1}^{n} \sum_{j=1}^{n}\left|\overline{p_{i j}}\right| \sum_{k=1}^{n}\left|p_{j k}\right|\left|z_{k}(t)\right| \\
& =-\beta \sum_{i=1}^{n} \lambda_{i}\left|z_{i}(t)\right|+l \sum_{i=1}^{n} \sum_{j=1}^{n} \sum_{k=1}^{n}\left|\overline{p_{j k}}\right|\left|p_{k i}\right|\left|z_{i}(t)\right| \\
& =\sum_{i=1}^{n}\left(-\beta \lambda_{i}+l \sum_{j=1}^{n} \sum_{k=1}^{n}\left|\overline{p_{j k}}\right|\left|p_{k i}\right|\right)\left|z_{i}(t)\right| \leq-(\beta \\
& \left.\cdot \min _{i}\left\{\lambda_{i}\right\}-l \max _{1 \leq k \leq n}\left\{\sum_{j=1}^{n}\left|\overline{p_{j k}}\right|\right\} \max _{1 \leq i \leq n}\left\{\sum_{k=1}^{n}\left|p_{k i}\right|\right\}\right) \\
& \cdot V(t, z(t))=-\left(\beta \min _{i}\left\{\lambda_{i}\right\}-l\left\|P^{-1}\right\|_{1}\|P\|_{1}\right) V(t \text {, } \\
& z(t))
\end{aligned}
$$

From (18), if $\beta / l>\|P\|_{1}\left\|P^{-1}\right\|_{1} / \min _{1 \leq i \leq n}\left\{\lambda_{i}\right\}$, inequality (8) can be satisfied almost everywhere. Therefore, according to Lemma 8 , when the multiagent system (10) with the control input (12) meets the conditions of Theorem 11, system (16) is Mittag-Leffler stable at the equilibrium point $z(t)=0$. It follows from $y(t)=P z(t)$ that $\lim _{t \rightarrow+\infty} y(t)=0$. This implies that $\lim _{t \rightarrow+\infty}\left|x_{i}(t)-x_{r}(t)\right|=0$ because $y_{i}(t)=$ $x_{i}(t)-x_{r}(t), i=1,2, \ldots, n$.

The proof is completed.

\section{Leader-Following Consensus for Double- Integrator Dynamics}

The leader-following consensus of fractional multiagent systems with double-integrator dynamics is investigated in this section.

Consider the multiagent system consisting of $n$ agents. The double-integrator dynamics of each follower is given by

$$
\begin{aligned}
{ }_{0} D_{t}^{\alpha} x_{i 0}(t) & =x_{i 1}(t), \\
{ }_{0} D_{t}^{\alpha} x_{i 1}(t) & =f\left(t, x_{i 0}(t), x_{i 1}(t)\right)+u_{i}(t), \\
i & =1,2, \ldots, n,
\end{aligned}
$$

where $\alpha \in(0,1), x_{i 0}(t)$ and $x_{i 1}(t)$ are the states for the $i$ th agent, $u_{i}(t)$ is the control input for the $i$ th agent, and $f\left(t, x_{i 0}(t), x_{i 1}(t)\right)$ is the intrinsic nonlinear dynamics for the $i$ th agent.

The virtual leader for multiagent system (19) is an isolated agent described by

$$
\begin{aligned}
& { }_{0} D_{t}^{\alpha} x_{r 0}(t)=x_{r 1}(t), \\
& { }_{0} D_{t}^{\alpha} x_{r 1}(t)=f\left(t, x_{r 0}(t), x_{r 1}(t)\right),
\end{aligned}
$$

where $x_{r 0}(t)$ and $x_{r 1}(t)$ are the states for the virtual leader.

The definition of the second-order leader-following consensus is given here, which will be used in the development of the next few sections.

Definition 12. The multiagent system (19) is said to achieve second-order leader-following consensus if its solution satisfies $\lim _{t \rightarrow+\infty}\left\|x_{i 0}(t)-x_{r 0}(t)\right\|=0, \lim _{t \rightarrow+\infty}\left\|x_{i 1}(t)-x_{r 1}(t)\right\|=$ $0, i=1,2, \ldots, n$, for any initial condition.

The following control input is considered to implement second-order leader-following consensus of multiagent system (19):

$$
\begin{aligned}
u_{i}= & -\sum_{j=1}^{n} a_{i j}\left[\left(x_{i 0}(t)-x_{j 0}(t)\right)+\beta\left(x_{i 1}(t)-x_{j 1}(t)\right)\right] \\
& -b_{i}\left[\left(x_{i 0}(t)-x_{r 0}(t)\right)+\beta\left(x_{i 1}(t)-x_{r 1}(t)\right)\right],
\end{aligned}
$$

where $a_{i j}(i, j=1,2, \ldots, n)$ is the $(i, j)$ th entry of the adjacency matrix $A \in R^{n \times n}$ associated with the undirected graph $\mathscr{G} ; b_{i}(i=1,2, \ldots, n)$ is a nonnegative real number that does not always equal 0 ; and $\beta$ is a nonnegative constant.

Let $B=\operatorname{diag}\left\{b_{1}, b_{2}, \ldots, b_{n}\right\}, \quad H=L+B$, and $A=$ $\left(\begin{array}{cc}0_{n \times n} & -I_{n} \\ H & \beta H\end{array}\right) \in R^{2 n \times 2 n}$, which is useful in the following statements, where $I_{n}$ is the $n \times n$ identity matrix and $\mathbf{0}_{n \times n}$ denotes the $n \times n$ zero matrix. Let matrix $\Delta$ be the Jordan canonical form of matrix $A$, which satisfies $\Delta=Q^{-1} A Q$, where $Q=$ $\left[q_{i j}\right] \in R^{2 n \times 2 n}$ and $Q^{-1}=\left[\overline{q_{i j}}\right] \in R^{2 n \times 2 n}$. 
Theorem 13. For system (19) with the control input (21), the second-order leader-following consensus is realized, if the fixed undirected graph $\mathscr{G}$ is connected and Assumption 3 holds, $\beta \geq$ $2 / \sqrt{\min _{1 \leq i \leq n}\left\{\lambda_{i}\right\}}, l<\min _{1 \leq i \leq 2 n}\left\{\delta_{i}\right\} /\|Q\|_{1}\left\|Q^{-1}\right\|_{1}$, where $\lambda_{i}$ is the ith eigenvalue of the matrix $H, i \in\{1,2, \ldots, n\}$, and $\delta_{i}$ is the ith eigenvalue of the matrix $A, i \in\{1,2, \ldots, 2 n\}$.

Proof. With the control input (21), system (19) can be rewritten as

$$
\begin{aligned}
& { }_{0} D_{t}^{\alpha} x_{i 0}(t)=x_{i 1}(t), \\
& { }_{0} D_{t}^{\alpha} x_{i 0}(t) \\
& =f\left(t, x_{i 0}(t), x_{i 1}(t)\right) \\
& \quad-\sum_{j=1}^{n} a_{i j}\left[\left(x_{i 0}(t)-x_{j 0}(t)\right)+\beta\left(x_{i 1}(t)-x_{j 1}(t)\right)\right] \\
& \quad-d_{i}\left[\left(x_{i 0}(t)-x_{r 0}(t)\right)+\beta\left(x_{i 1}(t)-x_{r 1}(t)\right)\right] .
\end{aligned}
$$

Noting that $y_{i 0}(t)=x_{i 0}(t)-x_{r 0}(t), y_{i 1}(t)=x_{i 1}(t)-$ $x_{r 1}(t), i=1,2, \ldots, n$, we have

$$
\begin{aligned}
& { }_{0} D_{t}^{\alpha} y_{i 0}(t)=y_{i 1}(t), \\
& { }_{0} D_{t}^{\alpha} y_{i 0}(t) \\
& =f\left(t, x_{i 0}(t), x_{i 1}(t)\right)-f\left(t, x_{r 0}(t), x_{r 1}(t)\right) \\
& \quad-\sum_{j=1}^{n} a_{i j}\left[\left(y_{i 0}(t)-y_{j 0}(t)\right)+\beta\left(y_{i 1}(t)-y_{j 1}(t)\right)\right] \\
& \quad-d_{i}\left(y_{i 0}(t)+\beta y_{i 1}(t)\right),
\end{aligned}
$$

for $i=1,2, \ldots, n$, which can be written in a vector form as

$$
\begin{aligned}
{ }_{0} D_{t}^{\alpha}\left(\begin{array}{c}
Y_{0}(t) \\
Y_{1}(t)
\end{array}\right)= & -A\left(\begin{array}{c}
Y_{0}(t) \\
Y_{1}(t)
\end{array}\right) \\
& +\left(\begin{array}{c}
0_{n \times 1} \\
F\left(t, Y_{0}(t), Y_{1}(t)\right)
\end{array}\right),
\end{aligned}
$$

where $Y_{k}(t)=\left[y_{1 k}(t), y_{2 k}(t), \ldots, y_{n k}(t)\right]^{T}(k=0,1)$, and $F\left(t, Y_{0}(t), Y_{1}(t)\right)=\left[f\left(t, y_{10}(t)+x_{r 0}(t), y_{11}(t)+x_{r 1}(t)\right)-\right.$ $f\left(t, x_{r 0}(t), x_{r 1}(t)\right), \ldots, f\left(t, y_{n 0}(t)+x_{r 0}(t), y_{n 1}(t)+x_{r 1}(t)\right)-$ $\left.f\left(t, x_{r 0}(t), x_{r 1}(t)\right)\right]^{T}$.

Suppose that $\delta_{i j}(i=1, \ldots, n ; j=1,2)$ are eigenvalues of the matrix $A$. From the results in [8], it is easy to obtain

$$
\begin{aligned}
& \delta_{i 1}=\frac{\lambda_{i}+\sqrt{\beta^{2} \lambda_{i}^{2}-4 \lambda_{i}}}{2}, \\
& \delta_{i 2}=\frac{\lambda_{i}-\sqrt{\beta^{2} \lambda_{i}^{2}-4 \lambda_{i}}}{2},
\end{aligned}
$$

where $\lambda_{i}$ is the $i$ th eigenvalue of the matrix $H, i \in\{1,2, \ldots, n\}$. When $\beta \geq 2 / \sqrt{\min _{1 \leq i \leq n}\left\{\lambda_{i}\right\}}$, all eigenvalues of the matrix $A$ are positive real numbers. So the matrix $\Delta$ is a diagonal

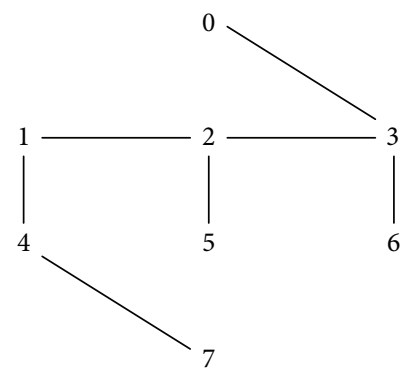

FIGURE 1: Interaction graph for a leader and 7 followers.

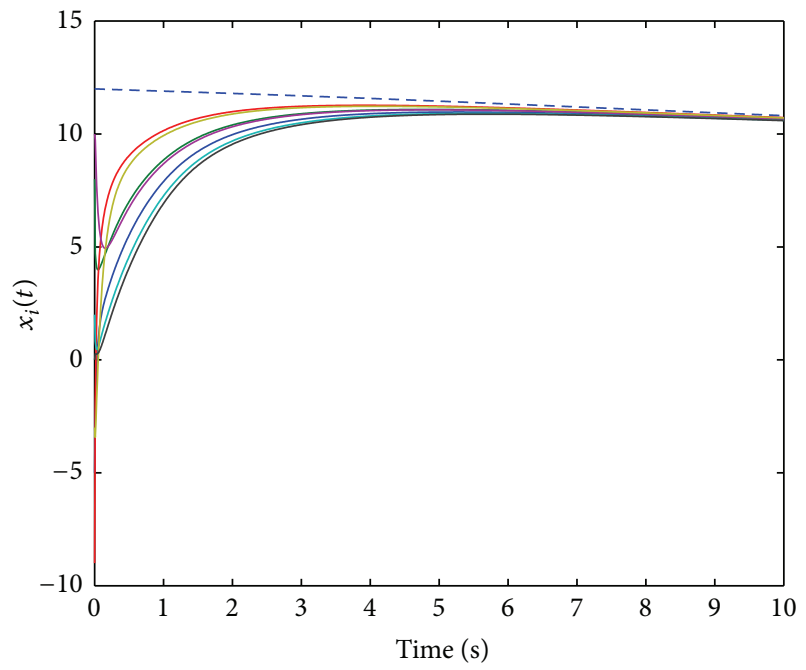

FIGURE 2: The states $x_{i}(t)(i=0,1, \ldots, 7)$ of all agents in system (11) and system (10) with $\alpha=0.9$.

positive definite matrix; that is, $\Delta=\operatorname{diag}\left\{\delta_{1}, \delta_{2}, \ldots, \delta_{2 n}\right\}, \delta_{i}>$ $0, i=1,2, \ldots, 2 n$.

Let $Z(t)=Q^{-1}\left(\begin{array}{l}Y_{0}(t) \\ Y_{1}(t)\end{array}\right)$, and (24) can be rewritten as

$$
{ }_{0} D_{t}^{\alpha} Z(t)=-\Delta Z(t)+Q^{-1}\left(\begin{array}{c}
0_{n \times 1} \\
F\left(t, Y_{0}(t), Y_{1}(t)\right)
\end{array}\right),
$$

where $Z(t)=\left[z_{1}(t), z_{2}(t), \ldots, z_{2 n}(t)\right]^{T}$. From Lemma 7 and Assumption 3, system (26) has a unique and continuous solution $Z(t)$. Then we prove the asymptotic stability of system (26).

Consider the Lyapunov function candidate constructed as

$$
V(t, Z(t))=\sum_{i=1}^{2 n}\left|z_{i}(t)\right|
$$

Obviously, the Lyapunov functional (27) satisfies inequality (7). According to Lemma 9 and (23), the following inequality holds almost everywhere: 


$$
\begin{aligned}
& { }_{0} D_{t}^{\alpha} V\left(t^{+}, Z\left(t^{+}\right)\right)=\sum_{i=1}^{2 n} D_{t}^{\alpha}\left|z_{i}\left(t^{+}\right)\right| \leq \sum_{i=1}^{2 n} \operatorname{sgn}\left(z_{i}(t)\right)_{0} D_{t}^{\alpha} z_{i}(t) \\
& =\sum_{i=1}^{2 n} \operatorname{sgn}\left(z_{i}(t)\right)\left[-\delta_{i} z_{i}(t)+\sum_{j=1}^{n} \overline{q_{i, j+n}}\left(f\left(t, y_{j 0}(t)+x_{r 0}(t), y_{j 1}(t)+x_{r 1}(t)\right)-f\left(t, x_{r 0}(t), x_{r 1}(t)\right)\right)\right] \\
& \leq \sum_{i=1}^{2 n}\left[-\delta_{i}\left|z_{i}(t)\right|+\sum_{j=1}^{n}\left|\overline{q_{i, j+n}}\right| l\left|y_{j 0}(t)+y_{j 1}(t)\right|\right]=\sum_{i=1}^{2 n}\left[-\delta_{i}\left|z_{i}(t)\right|+\sum_{j=1}^{n}\left|\overline{q_{i, j+n}}\right| l\left|\sum_{k=1}^{2 n} q_{j k} z_{k}(t)+\sum_{k=1}^{2 n} q_{j+n, k} z_{k}(t)\right|\right] \\
& \leq \sum_{i=1}^{2 n}\left[-\delta_{i}\left|z_{i}(t)\right|+l \sum_{j=1}^{n}\left|\overline{q_{i, j+n}}\right| \sum_{k=1}^{2 n}\left|q_{j k}+q_{j+n, k}\right|\left|z_{k}(t)\right|\right]=-\sum_{i=1}^{2 n} \delta_{i}\left|z_{i}(t)\right|+l \sum_{i=1}^{2 n} \sum_{j=1}^{n} \sum_{k=1}^{2 n}\left|\overline{q_{i, j+n}}\right|\left|q_{j k}+q_{j+n, k}\right|\left|z_{k}(t)\right| \\
& \quad=-\sum_{i=1}^{2 n} \delta_{i}\left|z_{i}(t)\right|+l \sum_{i=1}^{2 n} \sum_{j=1}^{n} \sum_{k=1}^{2 n}\left|\overline{q_{k, j+n}}\right|\left|q_{j i}+q_{j+n, i}\right|\left|z_{i}(t)\right| \leq-\min _{1 \leq i \leq 2 n}\left\{\delta_{i}\right\} \sum_{i=1}^{2 n}\left|z_{i}(t)\right| \\
& \quad+l \max _{1 \leq j \leq n}\left\{\sum_{k=1}^{2 n}\left|\overline{q_{k, j+n}}\right|\right\} \max _{1 \leq i \leq 2 n}\left\{\sum_{j=1}^{n}\left|q_{j i}+q_{j+n, i}\right|\right\} \sum_{i=1}^{2 n}\left|z_{i}(t)\right| \leq-\left(\min _{1 \leq i \leq 2 n}\left\{\delta_{i}\right\}-l\|Q\|_{1}\left\|Q^{-1}\right\|_{1}\right) \sum_{i=1}^{2 n}\left|z_{i}(t)\right| \\
& \quad=-\left(\min _{1 \leq i \leq 2 n}\left\{\delta_{i}\right\}-l\|Q\|_{1}\left\|Q^{-1}\right\| \|_{1}\right) V(t, Z(t)) .
\end{aligned}
$$

Hence, if $l<\min _{1 \leq i \leq 2 n}\left\{\delta_{i}\right\} /\|Q\|_{1}\left\|Q^{-1}\right\|_{1}$, inequality (8) can be satisfied almost everywhere. Therefore, according to Lemma 8, when the multiagent system (19) with the control input (21) meets the conditions of Theorem 13, system (26) is Mittag-Leffler stable at the equilibrium point $Z_{k}(t)=0(k=$ $0,1)$. It follows from $\left(\begin{array}{l}Y_{0}(t) \\ Y_{1}(t)\end{array}\right)=Q Z(t)$ that $\lim _{t \rightarrow+\infty} Y_{k}(t)=$ $0(k=0,1)$. This implies that $\lim _{t \rightarrow+\infty}\left|x_{i 0}(t)-x_{r 0}(t)\right|=0$ and $\lim _{t \rightarrow+\infty}\left|x_{i 1}(t)-x_{r 1}(t)\right|=0$ because $y_{i 0}(t)=x_{i 0}(t)-$ $x_{r 0}(t), y_{i 1}(t)=x_{i 1}(t)-x_{r 1}(t), i=1,2, \ldots, n$. Therefore, system (21) realizes the second-order leader-following consensus.

The proof of Theorem 13 is completed.

\section{Numerical Simulations}

Several numerical simulations are presented to verify the results of Theorem 11 proposed in Section 3 and Theorem 13 proposed in Section 4. We consider a group of 7 followers and a leader with an interaction graph given in Figure 1. Note that the interaction graph is connected. The corresponding Laplacian matrix is chosen as $a_{i j}=1$, if $\left(v_{j}, v_{i}\right) \in \mathscr{W}$, and $a_{i j}=0$ otherwise. It can be computed that the eigenvalues of the matrix $H$ are $0.07,0.382,0.6767,1.4080,2.6180,3.2982$, and 4.5472, respectively, and $\|P\|_{1}=2.4835,\left\|P^{-1}\right\|_{1}=2.3669$. Let $b_{i}=1$, if $i=3$, and $b_{i}=0$ otherwise.

Suppose that the nonlinear intrinsic dynamics of each agent in system (10) is described by $f\left(t, x_{i}(t)\right)=$ $(1 / 6) \sin \left(x_{i}(t)\right)$. Let the initial states of system (10) be $x(0)=[-5,8,-9,2,10,-3,0]^{T}$, where $x(0)=\left[x_{1}(0)\right.$, $\left.x_{2}(0), \ldots, x_{7}(0)\right]^{T}$. The nonlinear intrinsic dynamics of the virtual leader is given by $f\left(t, x_{r}(t)\right)=(1 / 6) \sin \left(x_{r}(t)\right)$. Let the initial states of system (11) be $x_{r}(0)=12$. We choose $\alpha=0.9$ and $\beta=15$ to satisfy the conditions of Theorem 11 .

Figure 2 shows the trajectories of the virtual leader in system (11) and the followers in system (10). It is easy to see from Figure 2 that the leader-following consensus is achieved in system (10).

We next present the simulation results to demonstrate Theorem 13 in Section 4. Suppose that the nonlinear intrinsic dynamics of each agent in system (19) is described by $f\left(t, x_{i 0}(t), x_{i 1}(t)\right)=(1 / 50) \sin \left(x_{i 0}(t)\right)+(1 / 50) \cos \left(x_{i 1}(t)\right)$. Let the initial states of system (19) be $X_{0}(t)=$ $[-5,8,-9,2,10,-3,0]^{T}, X_{1}(t)=[5,-9,2,-3,-7,1,-4]^{T}$. The nonlinear intrinsic dynamics of the virtual leader in system (20) is given by $f\left(t, x_{r 0}(t), x_{r 1}(t)\right)=$ $(1 / 50) \sin \left(x_{r 0}(t)\right)+(1 / 50) \cos \left(x_{\mathrm{r} 1}(t)\right)$. Let the initial states of system (20) be $x_{r 0}(0)=12, x_{r 1}(0)=6$. We choose $\alpha=0.9$ and $\beta=15$ to satisfy the conditions of Theorem 13 .

Figures 3 and 4 show the states of the virtual leader in system (20) and the followers in system (19). Figures 5 and 6 are trajectories of the leader and the followers from $t=0$ to $t=3$. It is easy to see from Figures 3 and 4 that the secondorder leader-following consensus is achieved in system (19).

\section{Conclusion}

In this paper, the leader-following consensus of fractional nonlinear multiagent systems is investigated via an undirected fixed interaction graph. Mittag-Leffler stability and the fractional Lyapunov direct method are firstly introduced into fractional multiagent systems to derive the main results. The control protocols are provided to extend the leader-following consensus for integer-order dynamics to fractional dynamics. 


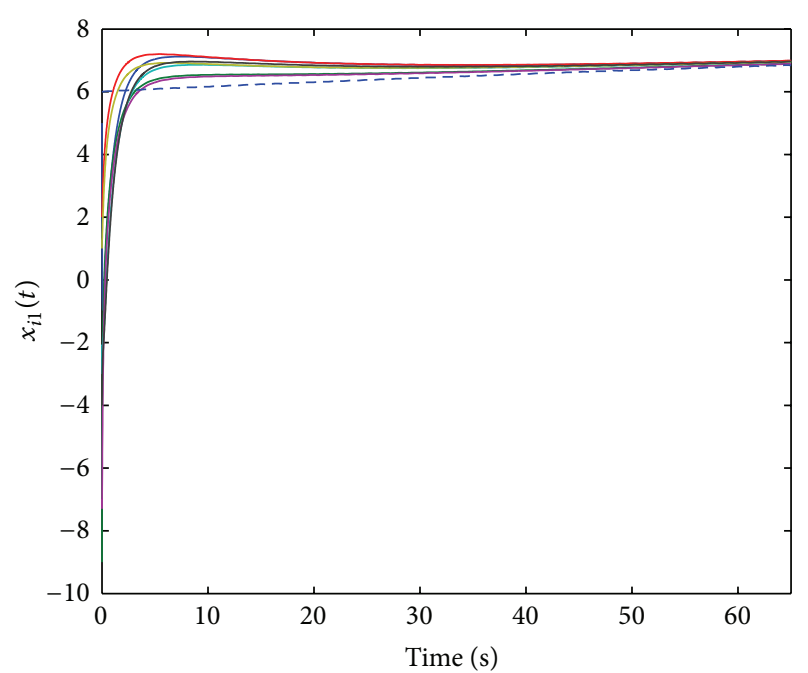

FIgURE 3: The states $x_{i 1}(t)(i=0,1, \ldots, 7)$ of all agents in system (22) and system (21) with $\alpha=0.9$.

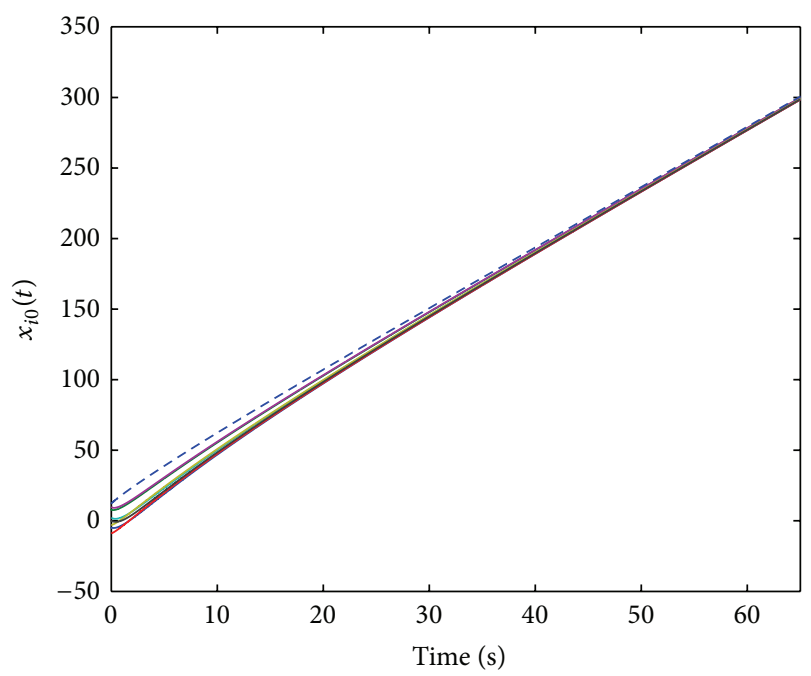

Figure 4: The states $x_{i 0}(t)(i=0,1, \ldots, 7)$ of all agents in system (22) and system (21) with $\alpha=0.9$.

The sufficient conditions are obtained such that the leaderfollowing consensus can be achieved in the systems with both single-integrator dynamics and double-integrator dynamics over a combination of the tools of Lyapunov method, matrix theory, and fractional calculus theory.

An effective and systematic approach to investigate the leader-following consensus problem of fractional systems is provided in this paper. Because most of the real-world complex networks are directed networks, such as World Wide Web and mobile communication network, we will study the leader-following consensus of fractional nonlinear multiagent systems in directed networks of agents in the future.

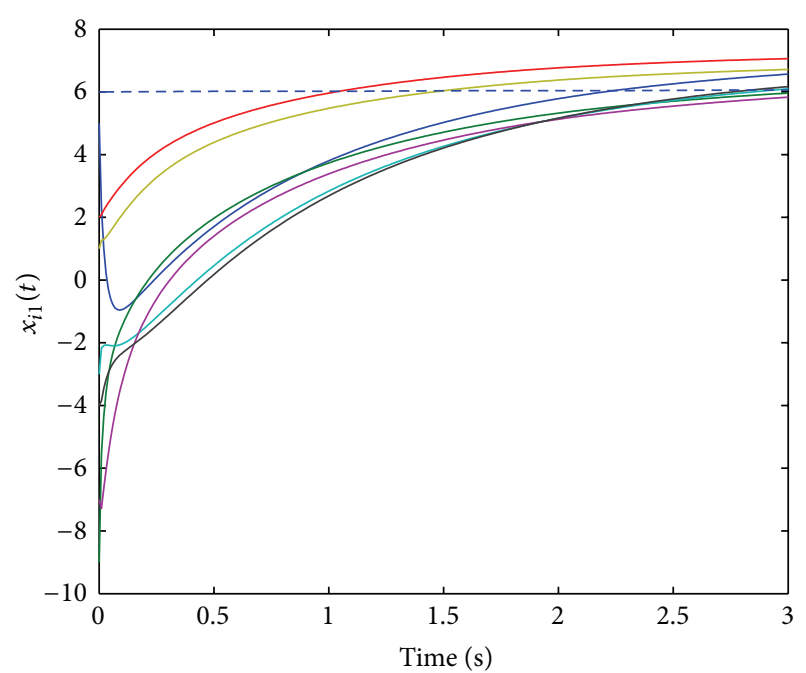

FIGURE 5: The states $x_{i 1}(t)(i=0,1, \ldots, 7)$ of all agents in system (22) and system (21) with $\alpha=0.9$.

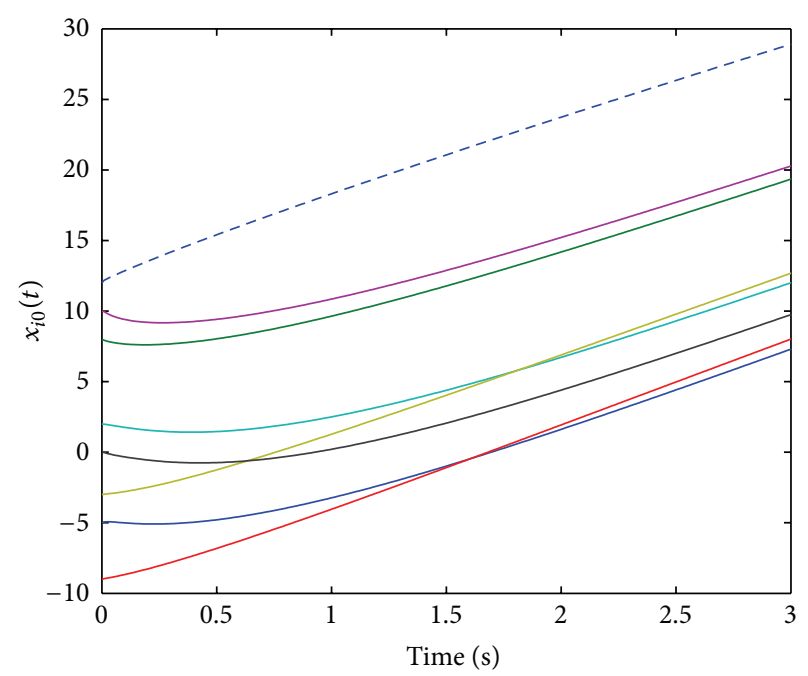

Figure 6: The states $x_{i 0}(t)(i=0,1, \ldots, 7)$ of all agents in system (22) and system (21) with $\alpha=0.9$.

\section{Conflict of Interests}

The authors declare that there is no conflict of interests regarding the publication of this paper.

\section{Acknowledgment}

This work is supported by the National Natural Science Foundation of China (no. 11371049).

\section{References}

[1] C. W. Reynolds, "Flocks, herds, and schools: a distributed behavioral model," Computer Graphics, vol. 21, no. 4, pp. 25-34, 1987. 
[2] T. Vicsek, A. Czirók, E. Ben-Jacob, I. Cohen, and O. Shochet, "Novel type of phase transition in a system of self-driven particles," Physical Review Letters, vol. 75, no. 6, pp. 1226-1229, 1995.

[3] Y.-P. Tian and C.-L. Liu, "Consensus of multi-agent systems with diverse input and communication delays," IEEE Transactions on Automatic Control, vol. 53, no. 9, pp. 2122-2128, 2008.

[4] J. Lv and G. Chen, "A time-varying complex dynamical network model and its controlled synchronization criteria," IEEE Transactions on Automatic Control, vol. 50, no. 6, pp. 841-846, 2005.

[5] W. Yu, G. Chen, and M. Cao, "Some necessary and sufficient conditions for second-order consensus in multi-agent dynamical systems," Automatica, vol. 46, no. 6, pp. 1089-1095, 2010.

[6] W. Yu, G. Chen, and M. Cao, "Consensus in directed networks of agents with nonlinear dynamics," IEEE Transactions on Automatic Control, vol. 56, no. 6, pp. 1436-1441, 2011.

[7] G. Wen, Z. Peng, A. Rahmani, and Y. Yu, "Distributed leaderfollowing consensus for second-order multi-agent systems with nonlinear inherent dynamics," International Journal of Systems Science, vol. 45, no. 9, pp. 1892-1901, 2014.

[8] Q. Song, J. Cao, and W. Yu, "Second-order leader-following consensus of nonlinear multi-agent systems via pinning control," Systems \& Control Letters, vol. 59, no. 9, pp. 553-562, 2010.

[9] Z. Meng, W. Ren, Y. Cao, and Z. You, "Leaderless and leaderfollowing consensus with communication and input delays under a directed network topology," IEEE Transactions on Systems, Man, and Cybernetics, Part B: Cybernetics, vol. 41, no. 1, pp. 75-88, 2011.

[10] Y. Cao, Y. Li, W. Ren, and Y. Chen, "Distributed coordination of networked fractional-order systems," IEEE Transactions on Systems, Man, and Cybernetics, Part B: Cybernetics, vol. 40, no. 2, pp. 362-370, 2010.

[11] H. Yang, X. Zhu, and K. Cao, "Distributed coordination of fractional order multi-agent systems with communication delays," Fractional Calculus and Applied Analysis, vol. 17, no. 1, pp. 23-37, 2014.

[12] Y. Cao and W. Ren, "Distributed formation control for fractional-order systems: dynamic interaction and absolute/ relative damping," Systems \& Control Letters, vol. 59, no. 3-4, pp. 233-240, 2010.

[13] W. Ren and Y. Cao, Distributed Coordination of Multi-Agent Networks: Emergent Problems, Models, and Issues, Springer, New York, NY, USA, 2010.

[14] I. Podlubny, Fractional Differential Equations, Academic Press, New York, NY, USA, 1999.

[15] A. Kilbas, H. Srivastava, and J. J. Trujillo, Theory and Applications of Fractional Differential Equations, Elsevier, 2006.

[16] Y. Li, Y. Chen, and I. Podlubny, "Stability of fractional-order nonlinear dynamic systems: lyapunov direct method and generalized Mittag-Leffler stability," Computers \& Mathematics with Applications, vol. 59, no. 5, pp. 1810-1821, 2010.

[17] S. Zhang, Y. Yu, and H. Wang, "Mittag-Leffler stability of fractional-order Hopfield neural networks," Nonlinear Analysis: Hybrid Systems, vol. 16, pp. 104-121, 2015. 


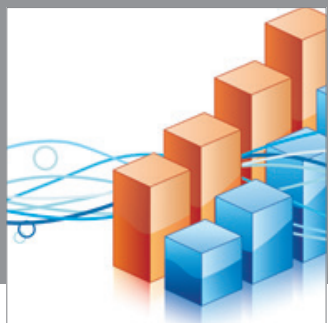

Advances in

Operations Research

mansans

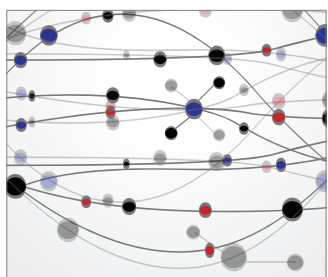

The Scientific World Journal
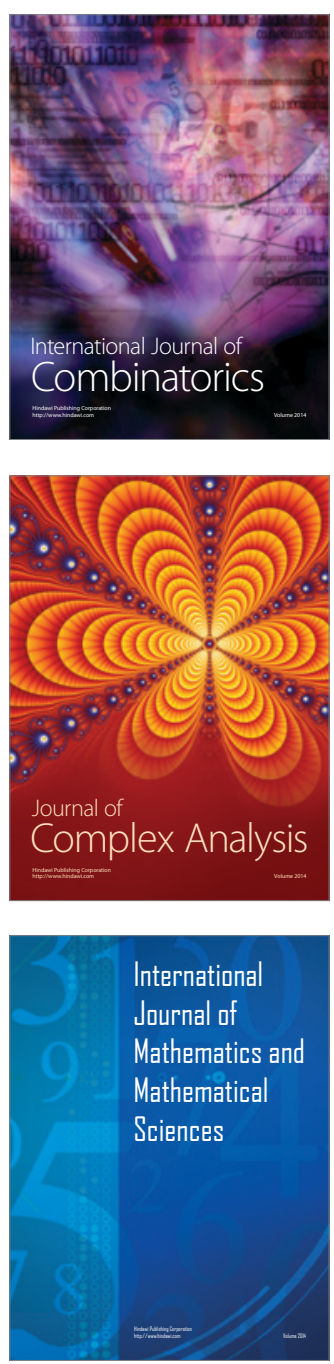
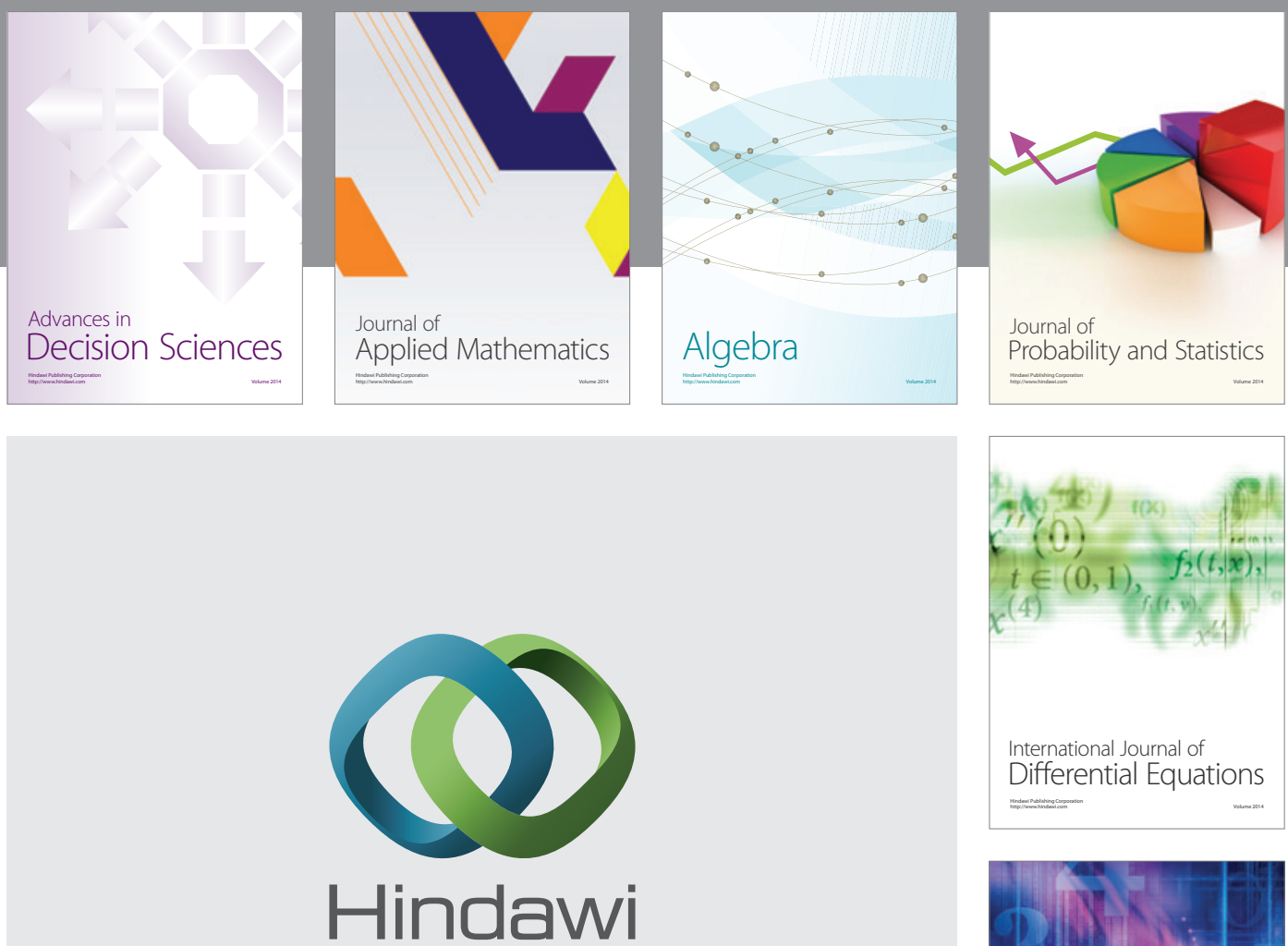

Submit your manuscripts at http://www.hindawi.com
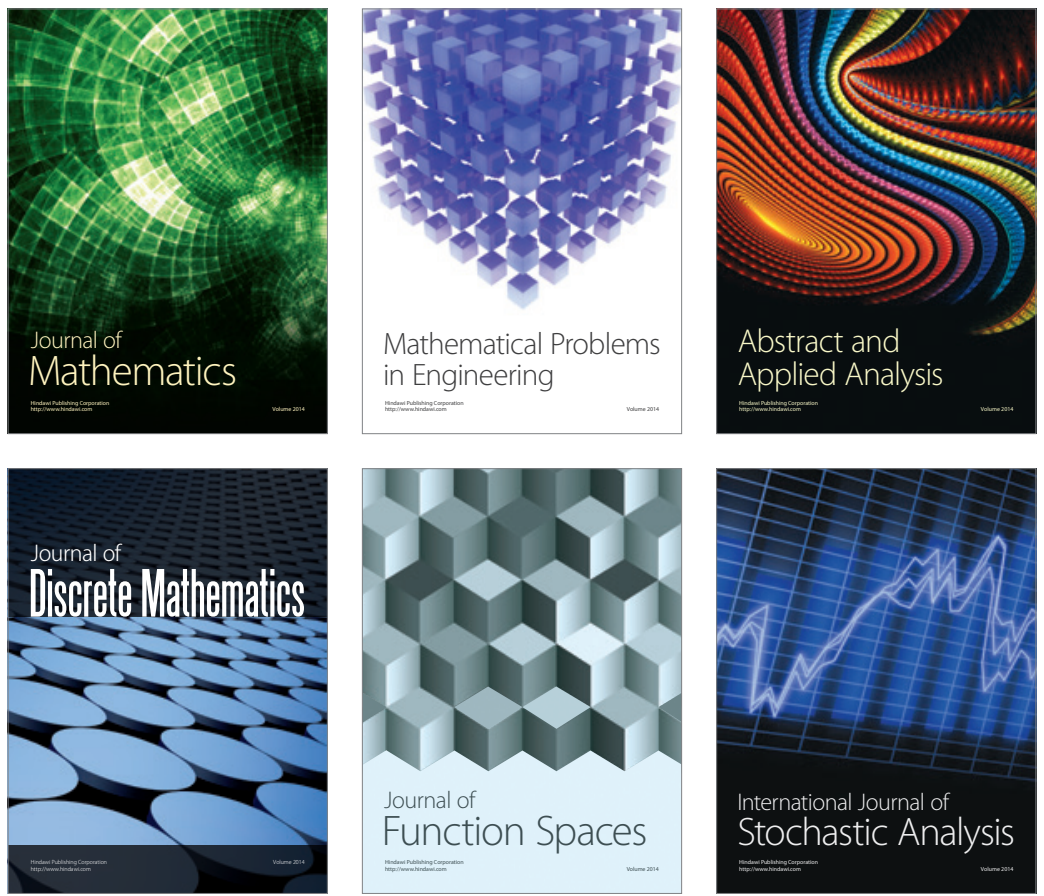

Journal of

Function Spaces

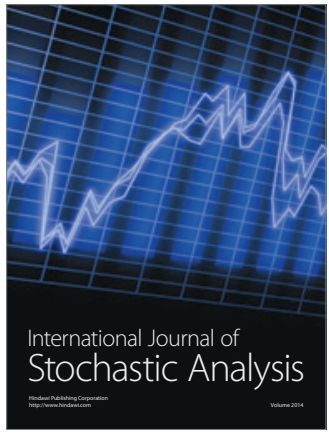

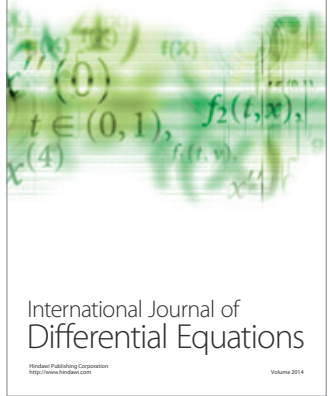
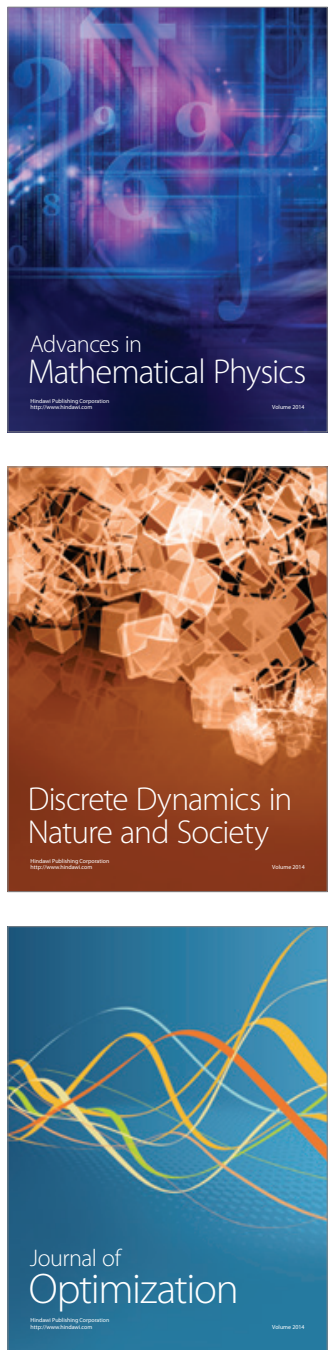